\title{
THE DIFFERENTIAL INVARIANTS OF A TWO-INDEX TENSOR
}

\author{
D. D. KOSAMBI
}

Riemannian geometry, based upon a metric form $d s^{2}=g_{i j} d x^{i} d x^{i}$, gives us the curvature tensor $R_{j k l}^{i}$ as the sole basic differential invariant of the space, and of the symmetric tensor $g_{i j}$. The general tensor $g_{i j}$ can be broken up into the sum of two irreducible components, namely the symmetric and antisymmetric portions defined respectively by $2 g_{(i j)}=g_{i j}+g_{j i}$ and $2 g_{[i j]}=g_{i j}-g_{j i}$. The latter disappears in constructing $d s^{2}$; but the general differential invariants of $g_{i j}$ must necessarily be composed of those derivable from $g_{(i j)}$ (the curvature tensor above), from $g_{[i j]}$, and a group of mixed invariants dependent upon both. It is proposed to investigate the general problem by use of a well known and easily proved fundamental lemma of the calculus of variations: The Euler equations derived from a variational principle are tensor-invariant under the group of transformations which leaves the original integral invariant. Actually the equations as directly obtained state that a certain covariant vector vanishes.

Given the tensor $g_{i j}\left(x^{1} \cdots x^{n}\right)$ we first introduce two (implicit) absolute parameters $u, v$, and construct the variational problem

$$
\delta \int g_{i j} x_{u}^{i} x_{v}^{j} d u d v=0 ; \quad x_{u}^{i}=\frac{\partial x^{i}}{\partial u}, \quad \text { and so on. }
$$

Only $x$-transformations will be allowed for the present. The Euler equations become

$$
\begin{gathered}
2\left\{g_{(i j)} x_{u v}^{j}+L_{j k i} x_{u}^{j} x_{v}^{k}\right\}=0 ; \\
L_{j k i}=\left(g_{i k, j}+g_{j i, k}-g_{j k, i}\right) / 2 ; \quad g_{i j, k}=\partial g_{i j} / \partial x^{k} .
\end{gathered}
$$

These $L_{i j k}$ must, therefore, have the law of transformation of Christoffel symbols of the first kind. In fact

$$
\begin{aligned}
L_{j k i} & =\left\{g_{(i k), j}+g_{(i j), k}-g_{(j k), i}\right\} / 2+\left\{g_{[i k], j}+g_{[j i], k}+g_{[k j], i}\right\} / 2 \\
& =\Gamma_{j k i}+\Omega_{j k i .}
\end{aligned}
$$

Here $\Gamma_{j k i}$ are precisely Christoffel symbols of the first kind associated with $g_{(i j)}$, and $\Omega_{j k i}$ is the fully covariant form of the Cartan torsion tensor. If now the discriminant $\left|g_{(i j)}\right| \neq 0$, we may construct $g^{(i j)}$ as usual to raise indices, and then obtain the coefficients of a general

Received by the editors February 26, 1948. 
affine connection:

$$
L_{j k}^{i}=g^{(i r)} L_{j k r}=\Gamma_{j k}^{i}+\Omega_{j k}^{i}
$$

with

$$
g_{(i j) \mid k} \equiv g_{(i j), k}-g_{(i r)} L_{j k}^{r}-g_{(r j)} L_{i k}^{r}=0 .
$$

That is, the covariant derivative of $g_{(i j)}$ with respect to the affine connection defined by (2) vanishes. This follows from the vanishing of the covariant derivative of $g_{(i j)}$ with respect to $\Gamma_{j k}^{i}$, and the antisymmetry of $\Omega_{i j k}$ in any pair of indices.

One may note in passing that $\Omega_{i j k}$ is the only basic differential invariant dependent upon $g_{[i j]}$ alone. Any differential invariant constructed from $g_{[i j]}$ must be based upon some form of tensor differentiation. If we take the covariant derivative with respect to any symmetric affine connection, we may write

$$
\begin{aligned}
\Omega_{i j k} & \equiv g_{[i j] \mid k}+g_{[j k] \mid i}+g_{[k i] \mid j} \\
& \equiv g_{[i j], k}+g_{[j k], i}+g_{[k i], j} .
\end{aligned}
$$

The antisymmetry of $g_{[i j]}$ and the symmetry of the connection coefficients make the latter cancel out of (5). The tensor $g_{[i j] \mid k}$ has only one other irreducible component besides $\Omega_{i j k}$, namely $g_{[i j] \mid k}+g_{[k j] \mid i}$ and this is clearly dependent upon the connection coefficients; hence, in this case, the remaining tensor-invariants are mixed. The condition $\Omega_{i j k}=0$ is necessary and sufficient for $g_{[i j]}$ to be a curl, that is, of form $\lambda_{i, j}-\lambda_{j, i}$, as is well known.

In the foregoing, however, we obtain no indication as to the intrinsic geometry of a purely antisymmetric $g_{i j}$, that is, when $g_{(i j)}=0$, though this is the only case where the integral in (1) is invariant under parameter transformation. This defect may again be remedied by utilizing another variational principle. We take a single absolute parameter $t$, and look for the geodesics of a metric of higher order (2) associated with $g_{i j} \dot{x}^{i} \ddot{x}^{j}$. But this is not an invariant under $x$-transformations, as $\ddot{x}^{j}$ is not a vector. We must therefore add to $\ddot{x}^{i}$ the terms $A_{j k}^{i} \dot{x}^{j} \dot{x}^{k}$, where $A_{j k}^{i}$ are as yet unspecified coefficients of affine connection symmetric, without loss of generality. Still further, we have to utilize the fact that a variational problem is unchanged by the addition of a perfect differential to the integral. If we integrate $g_{i j} \dot{x}^{i} \ddot{x}^{j} / 2$ by parts, we have left under the sign of integrationn $-g_{j i} \dot{x}^{i} \ddot{x}^{i} / 2-g_{i j, k} \dot{x}^{i} \dot{x}^{i} \dot{x}^{k} / 2$. The actual statement, therefore, is:

$$
\delta \int\left\{g_{[i j]} \dot{x}^{i} \ddot{x}^{j}+\left(-g_{i j, k} / 2+g_{i r} A_{j k}^{r}\right) \dot{x}^{i} \dot{x}^{j} \dot{x}^{k}\right\} d t=0 .
$$


The terms in parentheses are completely symmetric in all three lower indices $i j k$, and we may therefore take $A_{j k}^{r}=\Gamma_{j k}^{r}$, the Christoffel symbols associated with $g_{(i j)}$; then the terms in $g_{(i j)},{ }_{k} / 2$ cancel out with those in $g_{(i r)} A_{j k}^{r}$, leaving only $g_{[i r]} A_{j k}^{r} \dot{x}^{i} \dot{x}^{j} \dot{x}^{k}$. We may then drop the brackets in subscript, taking $g_{i j}=g_{[i j]}$ for we have finished with $g_{(i j)}$, which enters hereafter only through $\Gamma_{j k}^{t}$, its Christoffel symbols.

We make the further assumption that $\left|g_{i j}\right| \neq 0$ which implies that $n$, the number of dimensions of the space, is even. This is to allow raising and lowering of indices, along with a solution of the Euler equations explicitly for $d \ddot{x}^{i} / d t$. The equations themselves are

$$
2 g_{i j}\left\{d \ddot{x}^{j} / d t+3 \ddot{x}^{r} \dot{x}^{k} \gamma_{r k}^{j}+\beta_{k r s}^{j} \dot{x}^{k} \dot{x}^{r} \dot{x}^{s}\right\}=0,
$$

where

(a) $g_{i j}+g_{j i}=0$;

(b) $3 g_{i r} \gamma_{j k}^{r}=3 g_{i j, k} / 2+\left(g_{i k, j}+g_{j k, i}\right) / 2-H_{i j k}$;

(c) $2 g_{i l} \beta_{j k r}^{l}=g_{i(j, k, r)}-H_{i(j k, r)}+H_{r j k, i}$;

(d) with $H_{i j k}=g_{i r} \Gamma_{j k}^{r}+g_{j r} \Gamma_{k i}^{r}+g_{k r} \Gamma_{i j}^{r}$,

where the bracketed subscripts in $c$ denote only the completely symmetric portion. The tensor analysis of equations $d \ddot{x}^{i} / d t+\alpha^{i}(x, \dot{x}, \ddot{x}, t)$ $=0$ has been discussed elsewhere. The basic set of differential invariants in our present case would be, besides the vector $\dot{x}^{i}$ itself, the two tensors

$$
\begin{aligned}
& P_{r}^{i}=\alpha_{; r}^{i}-3\left(\frac{d}{d t} \gamma_{r}^{i}+\gamma_{k}^{i} \gamma_{r}^{k}\right) \\
& Q_{r}^{i}=\alpha_{, r}^{i}-\alpha_{; k}^{i} \gamma_{r}^{k}-\frac{d^{2}}{d t^{2}} \gamma_{r}^{i}+2 \gamma_{r}^{k} \frac{d}{d t} \gamma_{k}^{i}-2 \gamma_{k}^{i} \frac{d}{d t} \gamma_{r}^{k}+2 \gamma_{k}^{i k} \gamma_{l}^{k} \gamma_{r}^{l} ;
\end{aligned}
$$

where

$$
\begin{aligned}
\alpha^{i} & =3 \ddot{x}^{r} \dot{x}^{k} \gamma_{r k}^{i}+\beta_{k r s}^{i} \dot{x}^{k} \dot{x}^{r} \dot{x}^{s} \\
\alpha_{; r}^{i} & =\frac{\partial \alpha^{i}}{\partial \dot{x}^{r}}, \quad \text { and } \quad \gamma_{j}^{i}=\frac{1}{3} \frac{\partial \alpha^{i}}{\partial \ddot{x}^{j}}=\gamma_{j k}^{i} \dot{x}^{k} .
\end{aligned}
$$

The remaining (basic) differential invariants all vanish identically. Moreover, $\gamma_{j k}^{i}$ have the transformation law of coefficients of an affine connection, while there are three tensorial differential operators:

$$
\frac{\partial}{\partial \ddot{x}^{i}}, \quad \frac{\partial}{\partial \dot{x}^{i}}-2 \gamma_{i}^{r} \frac{\partial}{\partial \ddot{x}^{r}},
$$


and the third corresponding full covariant differentiation

$$
\begin{aligned}
\nabla_{r} u^{i} \equiv & u_{, r}^{i}-\gamma_{r}^{k}\left(\frac{\partial u^{i}}{\partial \dot{x}^{k}}-2 \gamma_{k}^{s} \frac{\partial u^{i}}{\partial \ddot{x}^{s}}\right) \\
& +\left\{2 \frac{d}{d t} \gamma_{r}^{k}-2 \gamma_{l}^{k} \gamma_{r}^{l}-\alpha_{i r}^{k}\right\} \frac{\partial u^{i}}{\partial \ddot{x}^{k}}+\gamma_{j r}^{i} u^{r} .
\end{aligned}
$$

We are primarily interested in those differential invariants obtained from (9)-(11) which are independent of $\dot{x}$ and $\ddot{x}$.

These invariants may be calculated very simply as follows. Wherever $\ddot{x}^{i}$ occurs, we add thereto the terms $\Gamma_{j k}^{i} \dot{x}^{j} \dot{x}^{k}$, and subtract the corresponding terms elsewhere in the same tensor. The tensor is thereby resolved into its invariant components, namely the coefficients of $\left(\ddot{x}^{i}+\Gamma_{j k}^{i} \dot{x}^{j} \dot{x}^{k}\right)$ and the homogeneous polynomials of various degree in $\dot{x}^{r}$.

The actual calculation is avoided almost entirely by the following considerations. It is known and easily proved that the connection coefficients $\gamma_{j k}^{i}$ in (8b) have the same law of transformation as Christoffel symbols of the second kind. Moreover, we have

$$
g_{i j, k}-g_{i r} \gamma_{j k}^{r}-g_{r j} \gamma_{i k}^{r}=0, \text { identically, }
$$

whence the covariant derivative of the basic tensor $g_{i j}$ vanishes with respect to its own "Christoffel symbols." Now the torsion, that is, the anti-symmetric portion, is seen to be

$$
g_{i r} \gamma_{[j k]}^{r}=\left(g_{i j, k}+g_{j k, i}-g_{i k, j}\right) / 2=\Omega_{i j k} / 6,
$$

as before. Since any tensor of the proper rank may be added to or subtracted from connection coefficients without affecting their transformation law, we have only to investigate invariants of the symmetric portion

$$
\gamma_{(j k)}^{r}=g^{i r}\left(g_{i j, k}+g_{i k, j}-H_{i j k}\right) / 3 .
$$

From these, again, one may eliminate $g_{i j, k}+g_{i k, j}$ by using their covariant derivatives with respect to $\Gamma_{j k}^{t}$, that is, $g_{i j \mid k}+g_{i k \mid j}$, which is seen to be the irreducible component of $g_{i j \mid k}$ mentioned before. We then have to investigate the curvature tensor formed by means of the symbols

$$
L_{j k}^{i}=\left\{\Gamma_{j k}^{r}+2 g^{i r}\left(\Gamma_{i k}^{s} g_{s j}+\Gamma_{i j}^{s} g_{s k}\right)\right\} / 3 .
$$

Choosing special coordinates in which $\Gamma_{j k}^{b}$ (but not their derivatives) vanish at a point, the tensor in question is seen to be 


$$
R_{j l k}^{i} / 3+2\left(g^{i r} g_{s j} R_{i l k}^{s}+g^{i r} g_{s k} R_{i l j}^{s}\right) / 3,
$$

where $R_{j k l}$ is the Riemann-Christoffel curvature tensor formed as usual from the $\Gamma_{j k}^{d}$. The derivation proves that the sole invariant depending only on the $g_{i j}$ is $\Omega_{i j k}$, as before.

To round out the discussion we have to consider other tensors of rank two, such as $g^{i j}$ and $g_{j}{ }^{i}$, as well as tensors of weight $p$ other than zero. The weight can be reduced to zero by division with a suitable power of the determinant except when the determinant vanishes, or $n p+2=0$, in which case nothing further can be done. Furthermore, we have the usual algebraic method of taking the normalized cofactors of the transposed matrix for associating a contravariant tensor $g^{i j}$ with a covariant $g_{i j}$. In this case, however, the geometry is associated with the variational principle $\delta \int g^{i j} \phi, i \phi, j d x^{1} \cdots d x^{n}=0$ and therewith a generalized Laplace equation. No new invariants are obtained. Finally, for the mixed tensor $g_{j}^{i}$, one may look for the extremum $\delta \int g_{j}^{i} \phi, i \dot{x}^{j} d t$ but it can be seen that this leads neither to a geometrical interpretation nor to differential invariants; though $\dot{x}^{i}$ may be eliminated from the resulting equations, the equations themselves cannot be solved explicitly for $\partial^{2} \phi / \partial x^{i} \partial x^{i}$. Thus, we obtain only the trivial cases (1) $g_{j}^{i}=\delta_{j}^{i}$ which is then an invariant of every affine connection, the integral above reducing to that of a perfect differential, hence independent of the path, and (2) the obvious invariant $g_{r, t}^{r}$.

BOMBAY, INDIA 